Atıf için / For Citation: O. Karaman, "Boosting performance of transfer learning model for diagnosis of COVID-19 from computer tomography scans", Süleyman Demirel Üniversitesi Fen Edebiyat Fakültesi Fen Dergisi, 16(1), 35-45, 2021.

\title{
Boosting Performance of Transfer Learning Model for Diagnosis of COVID-19 from Computer Tomography Scans
}

\author{
Onur KARAMAN ${ }^{* 1}$ \\ ${ }^{1}$ Akdeniz University, Vocational School of Health Services, Department of Medical Imaging Techniques, \\ 07070, Antalya, Turkey. \\ *corresponding author e-mail: onurkaraman@akdeniz.edu.tr
}

(Alinış / Received: 23.11.2020, Kabul / Accepted: 13.01.2021, Yayımlanma / Published: 27.05.2021)

\begin{abstract}
Early-stage rapid and accurate diagnosis of Corona Virus 2019 pneumonia is of great importance as a measure to the fight against the pandemic. Even if real-time reverse transcription-polymerase chain reaction (RT-PCR) test seems like a gold standard for determining COVID-19, the availability and the accuracy is still a challenge. Thus, alternative diagnostic techniques are required for controlling the spreading of the disease. Amongst the radiodiagnostic methods, the computer tomography (CT) technique is one of the most powerful candidates for screening COVID-19 pneumonia accurately. In this study, it is aimed to develop a reliable transfer learning-based Convolutional Neural Networks (CNN) model tailored to detect the COVID-19 from chest CT scans with high accuracy and sensitivity to help to accelerate the application of the required treatment and taking of measures. The CT scan dataset used in the training process of the CNN model was obtained from "SARS-CoV-2 CT-Scan Dataset". This dataset contains 1252 CT scans for positive COVID-19 cases and 1230 CT scans for the non-COVID-19 cases, all data have been collected from real patients from hospitals in Sao Paulo, Brazil. ResNet18, ResNet50, VGG16, AlexNet, and SqueezeNet1_1 architectures were re-trained to extract COVID-19 classes by transfer learning. The highest classification performance parameters were obtained for ResNet50 architecture and were calculated as $99.80 \%$ accuracy, $99.61 \%$ precision, and $100.00 \%$ sensitivity. The activation maps were created to highlight the crucial areas of the CT images and improve causality and intelligibility. As a result of this study, it has been shown that the developed transfer learning model can be utilized for reliable clinical diagnosis of COVID-19 cases from CT images to accelerate the triaging and save critical time for disease control as well as assisting the radiologist to validate their initial diagnosis.
\end{abstract}

Key words: Corona virus (COVID-19), SARS-CoV-2 CT-Scan Dataset, Convolutional neural network (CNN), Transfer learning, Computer tomography

\section{Bilgisayarlı Tomografi Görüntülerinden COVID-19 Teşhisi İçin Geliştirilen Transfer Öğrenim Modelinin Performansının Artırılması}

Özet: COVID-19 pnömonisinin erken aşamada hızlı ve doğru teşhisi, pandemiyle mücadelede bir önlem olarak büyük önem taşımaktadır. Gerçek zamanlı ters transkriptaz-polimeraz zincir reaksiyonu (RT-PCR) testi, COVID-19'u belirlemek için altın bir standart olarak kullanılsa da, kullanılabilirliği ve doğruluğu açısından değerlendirildiğinde eksiklikleri mevcuttur. $\mathrm{Bu}$ nedenle, hastalığın yayılmasını kontrol etmek için alternatif teşhis teknikleri gereklidir. Radyodiyagnostik yöntemler arasında, bilgisayarlı tomografi (BT) tekniği COVID-19 pnömonisini doğru bir şekilde teşhis etmek için en güçlü adaylardan biridir. Bu çalışmada, gerekli tedavinin uygulanmasını ve önlemlerin alınmasını hızlandırmaya yardımcı olmak için 
gögüs BT taramalarından COVID-19'u yüksek doğruluk ve hassasiyetle tespit etmek üzere uyarlanmış, aktarımı öğrenmeye dayalı güvenilir bir evrişimsel sinir ağı $(\mathrm{CNN})$ modelinin geliştirilmesi amaçlanmıştır. CNN modelinin eğitim sürecinde kullanılan BT görüntüleri veri seti "SARS-CoV-2 CT-Scan Veri Seti”nden elde edilmiştir. Bu veri kümesi, pozitif COVID-19 vakası için 1252 BT görüntüsü ve COVID-19 olmayan vaka için 1230 BT görüntüsü, toplamda 2482 BT görüntüsü içerir, tüm veriler Sao Paulo, Brezilya'daki hastanelerdeki gerçek hastalardan toplanmıştır. ResNet18, ResNet50, VGG16, AlexNet ve SqueezeNet1_1 mimarileri, transfer öğrenimi ile COVID-19 sınıflarını belirlemek için yeniden eğitildi. En yüksek sınıflandırma performans parametreleri ResNet50 mimarisi için elde edilmiş ve \% 99,80 doğruluk,\% 99,61 kesinlik ve\% 100,00 duyarlılık olarak hesaplanmıştır. BT görüntülerinin önemli alanlarını vurgulamak ve nedensellik ve anlaşılırlığı geliştirmek için aktivasyon haritaları oluşturuldu. Geliştirilen transfer öğrenme modeli, triyajı hızlandırmak ve hastalık kontrolü için kritik zamandan tasarruf etmek ve radyoloğun ilk tanılarını doğrulamasına yardımcı olmak için BT görüntülerinden COVID-19 vakalarının güvenilir klinik teşhisinde kullanılabilir.

Anahtar kelimeler: Corona virüsü (COVID-19), SARS-CoV-2 CT-Scan Veri Seti, Evrişimsel sinir ağı $(\mathrm{CNN})$, Transfer öğrenimi, Bilgisayarlı tomografi

\section{Introduction}

The new coronavirus disease (COVID-19) declared as a pandemic by World Health Organization (WHO) has caused a major impact on not only the health but also the economy. Acute respiratory disorder, fever, cough, sore throat, muscle pain, and shortness of breath are the main manifests of the disease [1,2]. The researches have been demonstrated that the early-stage diagnosis of the COVID-19 infected patients is the most crucial step in both fight against the pandemic and decreasing the death rate [3-6]. The diagnosis procedure of COVID-19 disease mainly follows these steps; i) clinical symptoms; ii) epidemiological history, and iii) viral nucleic acid test or Computer Tomography (CT) images [7-9]. Even though the RT-PCR test is mainly accepted as a standard diagnostic method, the lack of test kits especially in hyper-affected areas, lower sensitivity, specificity and reproducibility of the test kit are among the main disadvantages $[1,10,11]$. Furthermore, not only it is hard to detect the COVID-19 infected patient in early-stage with RT-PCR test but also it is quite a time-consuming process comparing to radiological imaging techniques $[9,10]$. Radiological imaging techniques especially CT imaging has been shown to be effective tools in detection, quantification, and follow-up of COVID-19 [6-9]. In these techniques, the obtained radiological images need to be reported by radiologist to detect the visual pattern associated with the SARS-CoV-2 viral infection. However, sometimes it is not easy to distinguish COVID-19 pneumonia from other types of pneumonia since they are similar to each other and sometimes certain manifestations may overlap with other lung infections [9]. Thus, recently most of the researchers have devoted considerable attention toward the developing deep learning models to identify the hallmarks of disease that might be difficult for visual recognition $[1,6,7,12,13]$. It was stated that almost all patients infected by COVID-19 had characteristic CT features during the disease [14-16]. Since it provides rich pathological information about the infection region and their longitudinal changes, subsequent CT scans every 3-5 days in follow up assessment of hospitalized COVID-19 patients are recommended to evaluate the therapeutic responses [17]. Thus, a rapid automated tool for monitoring COVID-19 infection urgently needed in the onsite applications for quantitative assessment of the disease. By implementation of deep learning models in the healthcare system, both the quality of healthcare and the welfare of the patients can be improved and the diagnostic 
can be more effective [18]. Additionally, it may be possible to lower healthcare costs by means of utilizing artificial intelligence and machine learning models in such systems.

Screening of the disease by means of deep-learning algorithms from radiodiagnostic images is revolutionary step in the pandemic period. Bearing this in mind, in this study, a reliable transfer learning based Convolutional neural network (CNN) model tailored to detect the COVID-19 from chest CT scans with high accuracy and sensitivity was developed to help the accelerating the application of the required treatment and taking of measures. Different architectures including ResNet18, ResNet50, VGG16, AlexNet, and SqueezeNet 1_1 were re-trained in the transfer learning process to boost the performance of the model, and according to calculated performance parameters, the architecture with the best performance parameters was determined. Since the developed model performs auto-contouring of infected regions by means of activation maps, it will assist the clinicians in improved, rapid, and accurate scanning and diagnostic in their daily routine work, and the workload of clinicians will be significantly reduced.

\section{Material and Method}

\subsection{Details of datasets}

In this work, datasets collected from "SARS-CoV-2 CT-Scan Dataset" resource available at https://www.kaggle.com/plameneduardo/sarscov2-ctscan-dataset_[14]. The dataset is composed a total of 2482 CT scans, collected from different hospitals in Sao Paulo, Brazil, while 1252 of which is for COVID-19-infected patients and $1230 \mathrm{CT}$ scans for non-COVID-19 infected patient, but that have other pulmonary diseases. Figure 1. illustrates the detailed number of patients composing the dataset. In this study, the collected data were classified as non-COVID-19 pneumonia and COVID-19 pneumonia for further processes. $80 \%$ of each COVID-19 positive and non-COVID-19 CT images were used for training and $20 \%$ of it was used for validation sets.

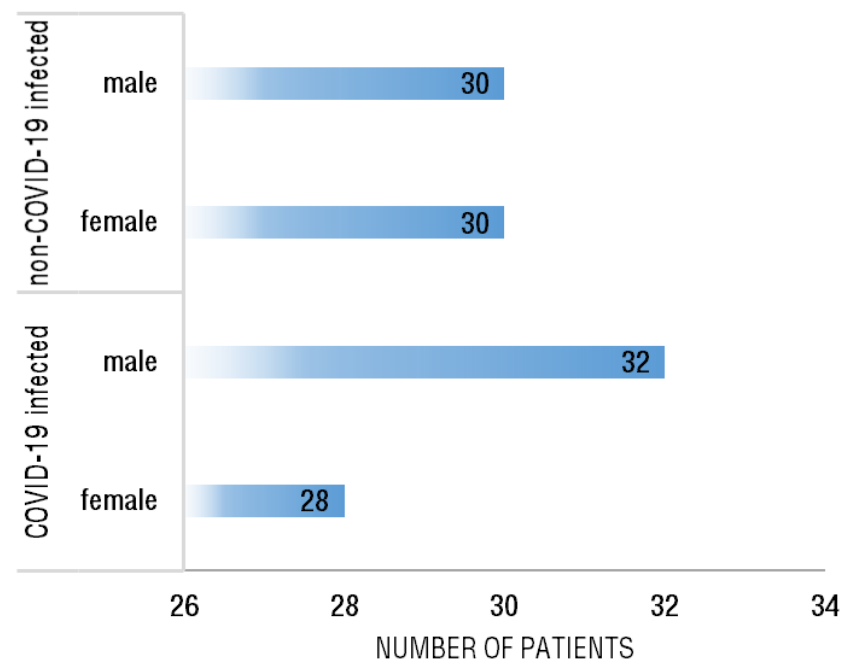

Figure 1. The detailed number of patients composing the SARS-CoV-2 CT-Scan Dataset [14]

\subsection{Transfer learning and optimization}

The transfer learning and optimization of the model was conducted on a personal computer (Ryzen 51600 CPU, 16GB DDR4 RAM, Nvidia RTX 2080 Ti Graphics Card). Fastai [19] framework was used to develop the model. Residual Neural Network (ResNet) [20], Visual Geometry Group (VGG) [21], SqueezeNet, and AlexNet [22] 
architectures, which have already been trained by the ImageNet [23] database, were retrained to distinguish COVID-19 pneumonia and non-COVID-19 pneumonia by transfer learning. During transfer learning studies, firstly, the convolution layers of ResNet18, ResNet50, VGG16, SqueezeNet1_1 and AlexNet architectures were frozen and only fully connected layers were re-trained. The learning rate was explored from $10^{-6}$ to 1.0 over 170 iterations for all architectures, and the learning rate values for each architecture were determined same as $10^{-2}$ and they were trained in a total of 60 epochs.

The VGG Net is developed a result of an idea of much deeper networks with much smaller filters. There are three types of VGG Net architectures including VGG-11, VGG-16, and VGG-19 and these networks contain 11, 16 and 19 convolutional and fully connected layers. The main base element of ResNet is the residual blocks. In ResNet, all these residual blocks are stacked together very deeply. It prevents the distortion that occurs as the network gets deeper and more complex. In addition, bottleneck blocks are used to make training faster in the ResNet model. Another thing with this very deep architecture is that it is enabling up to 150 layers deep of this. AlexNet architecture consists of 5 convolutional layers, 3 max-pooling layers, 2 normalization layers, 2 fully connected layers, and 1 softmax layer. Each convolutional layer consists of convolutional filters and a nonlinear activation function ReLU. AlexNet overall has 60 million parameters. On the otherhand, SqueezeNet is a smaller network that was designed as a more compact replacement for AlexNet. It has almost 50 times fewer parameters than AlexNet, yet it performs 3 times faster. The SqueezeNet architecture is comprised of "squeeze" and "expand" layers [14].

In fine-tuning process, the frozen convolutional layers were re-trained by unfreezing the layers. In the backpropagation process, the "stochastic gradient descent with restarts" [24] technique was used to determine the minimum points on the loss function. As the iterations progress, the learning rate value has been updated by repeating with the "cosine annealing" method and by expanding the period. Thanks to the dynamically updated learning rate, the minimum points on the "loss function" can be determined more accurately. The limits of the learning rate values should be determined to apply the "stochastic gradient descent with restarts" technique to the architectures trained at the first stage of transfer learning process. To determine the limit of the learning rate values, the learning rate exploration process of unfrozen networks was repeated and the appropriate learning rate interval to be studied was determined. The learning rate limits of the cosine annealing algorithm used in the fine-tuning process determined differently for all architectures and ranging between $10^{-6}$ and $10^{-3}$.

\subsection{Activation maps}

Gradient-weighted Class Activation Mapping (Grad-CAM) method helping to improve causality and intelligibility was used to create activation maps that highlights the crucial areas of the CT images [25,26]. In the Grad-CAM method, the gradients of the CT scans flowing into the final convolutional layer to produce a rough localization map in which the important areas are highlighted is used. It has been reported that the deeper presentations in a convolutional neural network capture upper-level visual constructs $[27,28]$. Additionally, the convolutional layers able to keep spatial information which is lost in fully-connected layers. Thus, it can be expected the last convolutional layers to have the perfect concurrence between high-level semantics and detailed spatial information. The semantic class-specific information in the image are tried to find by 
the neurons (i.e. object parts). Grad-CAM uses the gradient information flowing into the last convolutional layer to assign significance values to each neuron.

\subsection{Performance evaluation criteria}

The accuracy, recall (sensitivity) and precision parameters are the main parameters to determine the performance of the model. In confusion matrix there is four possible outcomes including true positive (TP), true negative (TN), false positive (FP), false negative (FN). When the case is actually positive, if it is classified as positive it is labeled as TP, in contrast, if it is classified as negative it is labeled as FN. Similarly, in the case of negative, if the case is classified as negative it is TN, if is classified as positive it is labelled as FP [29]. To evaluate the performance of the tailored convolutional neural networks the accuracy, sensitivity and precision coefficients were determined as followings:

$$
\begin{gathered}
\text { Accuracy } \%=\left(\frac{T P+T N}{T P+T N+F P+F N}\right) \times 100 \\
\text { Sensitivity } \%=\left(\frac{T P}{T P+F N}\right) \times 100 \\
\text { Precision } \%=\left(\frac{T P}{T P+F P}\right) \times 100
\end{gathered}
$$

\section{Results}

The training process consists of three basic stages as followings; i) different architectures including ResNet, VGG, AlexNet, and SqueezeNet were examined and all convolutional layers were frozen and trained $i$ ) the fine-tuning process was performed for each architecture iii) the inference results of the activation map were evaluated, which establishes a bridge between clinicians and artificial intelligence for understanding.

\subsection{Transfer learning and optimization}

In the first stage of transfer learning, fully connected layers of ResNet, VGG, AlexNet, and SqueezeNet architectures, previously trained by ImageNet datasets, were re-trained and structured to distinguish between COVID-19 pneumonia and non-COVID-19 pneumonia. All training conditions were set as a batch size of 32 and the learning rate of 1e-2. Then, the fine-tuning on ResNet18, ResNet50, VGG16, AlexNet, and SqueezeNet1_1 architectures was performed to improve the performance criteria. The learning rate exploration process must be repeated on the unfrozen model and the maximum and minimum learning rate limits to be used must be determined for applying SGDR to the model [24]. These determined learning rate values were used as maximum and minimum learning rate values in the cosine annealing process.

As a result of the total of 60 epoch training process, validation loss, validation accuracy, recall and precision metrics were calculated after each epoch and the results were plotted as depicted in Figure 2. Validation loss values for nearly all architectures except 
AlexNet appeared to decrease exponentially and had a stable structure by reaching saturation.
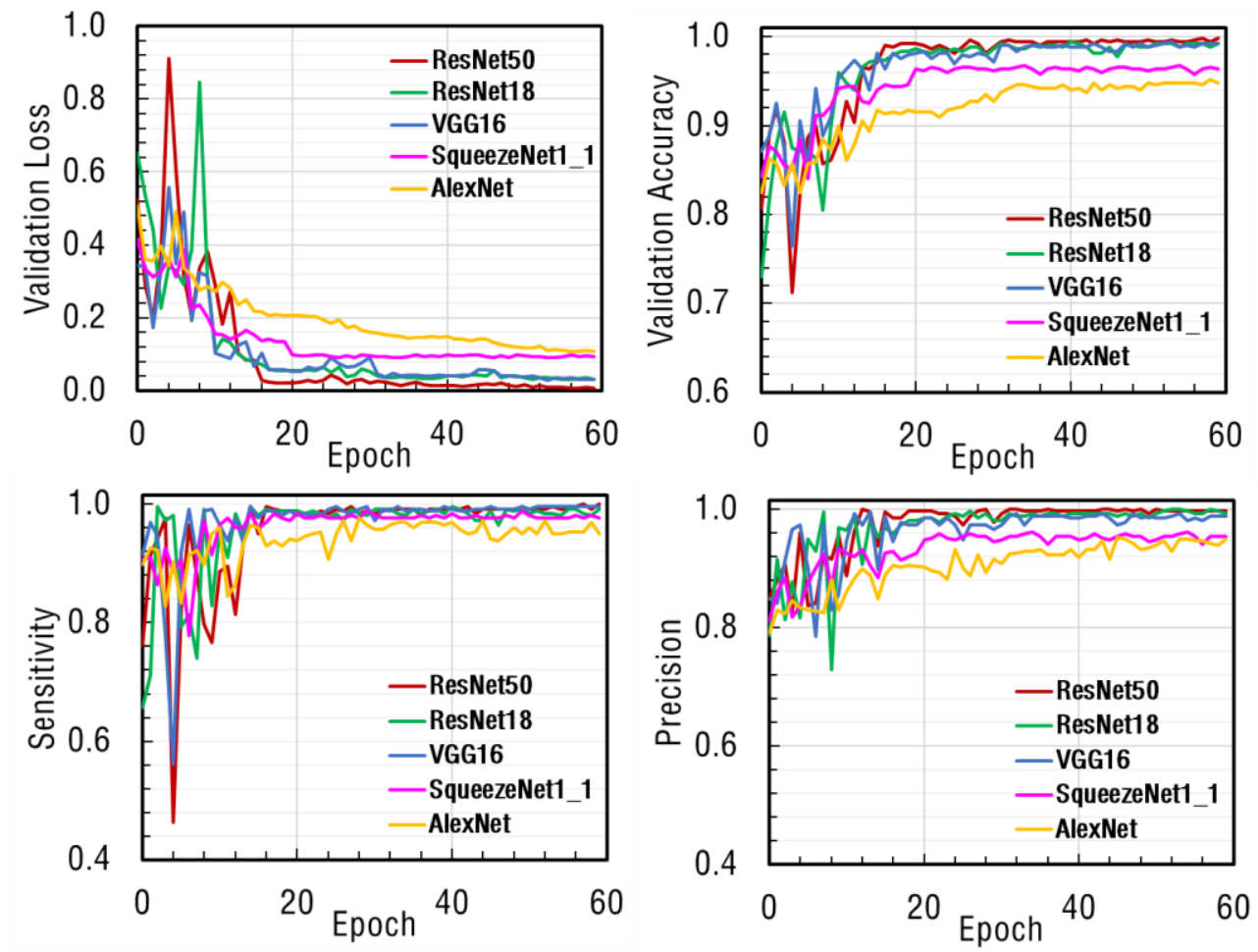

Figure 2. The comparison of performance graphs of different metrics for ResNet50, ResNet18, VGG16, SqueezeNet1_1, and AlexNet architectures

According to the information obtained from the graphs, the confusion matrices were calculated at the points where the models perform at maximum, and it was seen that the ResNet50 architecture performs better than other architectures with the following graphs (Figure 3). 

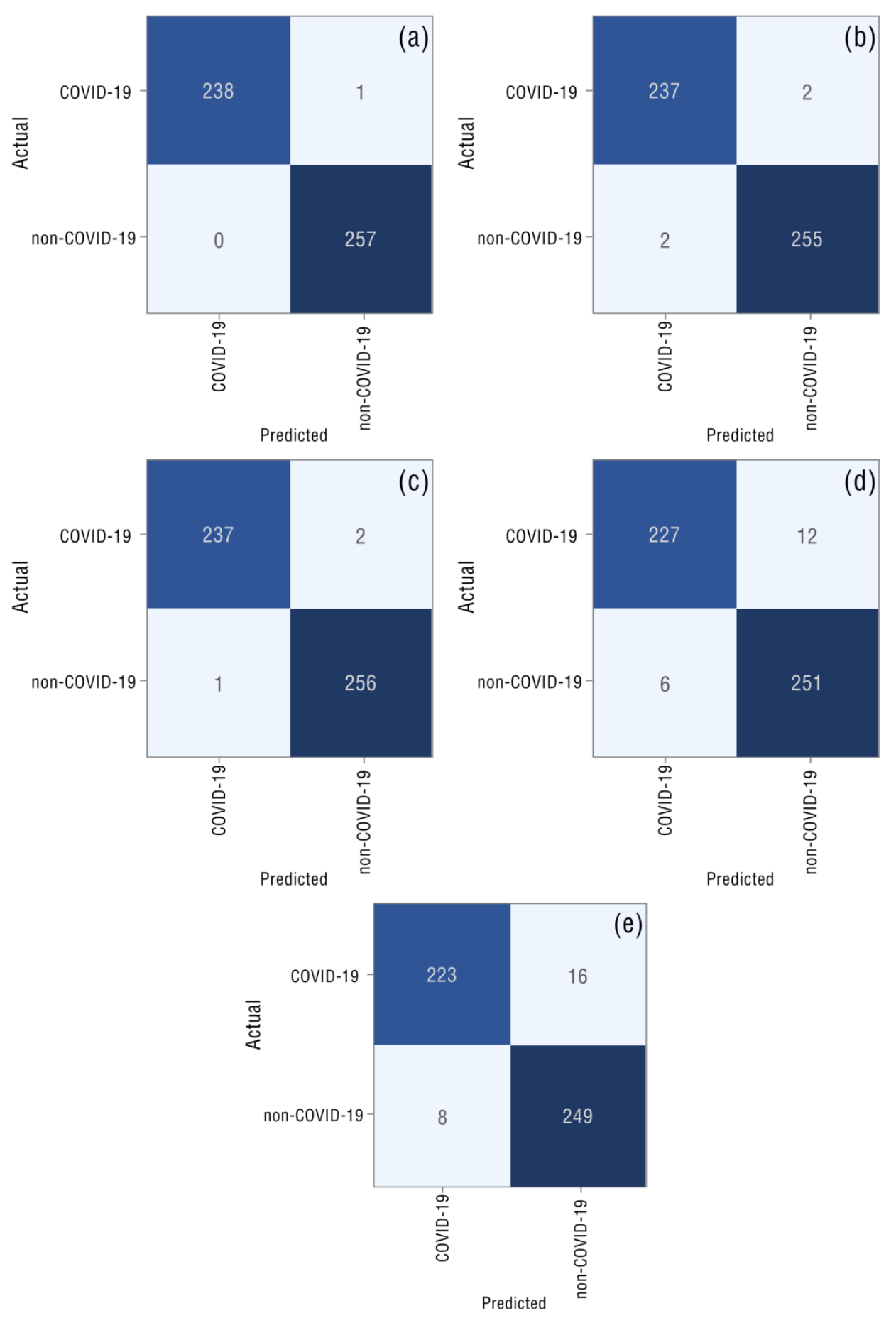

Figure 3. Confusion matrix of developed model for (a) ResNet50 (b) ResNet18 (c) VGG-16 (d) SqueezeNet1_1 (e) AlexNet architectures

The calculated results of the fine tuning process of transfer learning were tabulated in Table 1. As can be seen from the chart, ResNet50 architecture shows the best performance in accuracy, sensitivity and precision metrics. Additionally, it should be noted that all trained networks presented high sensitivity values thanks to fine tuning. Unlike other architectures, ResNet50 architecture was achieved the highest performance since deep networks can be created without encountering overfitting problems in this architecture thanks to its "skipped connections" content. 
Table 1. Comparison of performance metrics of developed transfer learning based CNN model for different architectures

\begin{tabular}{lccc}
\hline Architecture & Accuracy & Precision & Sensitivity \\
\hline ResNet50 & 99.80 & 99.61 & 100.00 \\
ResNet18 & 99.19 & 99.22 & 99.22 \\
VGG16 & 99.19 & 98.84 & 99.61 \\
AlexNet & 94.76 & 94.94 & 94.94 \\
SqueezeNet1_1 & 96.37 & 95.44 & 97.67 \\
\hline
\end{tabular}

In Table 2, a comparison of the accuracy and sensitivity values of some artificial intelligence models developed for the diagnosis of COVID-19 from CT images was presented. The model developed in this study is among the models with the highest performance compared to other studies on the COVID-19 diagnosis with artificial intelligence supported models from CT images in the literature. In this study, despite the use of the same database with Soares et al., an approximately $2 \%$ increase in accuracy value is noteworthy. The main reason for this is the fine-tuning process on the ResNet50 architecture used in the $\mathrm{CNN}$ model based on transfer learning.

Table 2. Summary of deep learning-based models for identifying COVID-19 from chest CT images

\begin{tabular}{cccc}
\hline Reference & Model & Accuracy \% & Sensitivity \% \\
\hline Current work & $\begin{array}{c}\text { Transfer Learning } \\
\text { based CNN } \\
\text { Explainable deep } \\
\text { learning } \\
\text { 2D and 3D deep } \\
\text { learning }\end{array}$ & 99.8 & 100.0 \\
{$[14]$} & $\begin{array}{c}\text { Transfer learning } \\
\text { based deep } \\
\text { learning }\end{array}$ & 89.6 & 95.5 \\
{$[9]$} & 82.9 & 98.2 \\
\hline
\end{tabular}

\subsection{Activation map}

Since radiodiagnosis takes an important role in the detection of early-stage COVID-19 cases and taking further measures, it is crucial to train the CNN model to detect more sensitively of COVID-19 positive lung. Thus, in the last part of the study, the original labels of the radiographs of COVID-19 cases confirmed positive by the RT-PCR test and the predictions errors of the CNN model outputs were evaluated by an expert radiologist to confirm model robustness. The developed model is capable to determine the pathognomonic findings for COVID-19 including ground-glass opacities, consolidation areas, and nodular opacities. In the chest CT images of COVID-19 infected patients, bilateral, lower lobe and peripheral involvement can be observed. Thus, the developed model able to detect the localization of these to identify the early stages of COVID-19 cases [13]. The activation mapping was applied to the CT scans by Grad-CAM algorithm so that radiologists could easily diagnose accordingly (Figure 4.). The original radiograph, the activation map calculated by the Grad-CAM algorithm were presented in Figure 4. where the CT cross-section view is on the left and the CT image in color with the $7 \times 7$ activation map is located on the right. The regions where the model was activated were colored from the lowest activation to the highest activation from blue to red. As it can be seen on these validation data created with the 
activation map, sections containing the COVID-19 findings have been colored, in this way causality and explainability were enhanced in the study [13]. It was concluded that the developed model could be used in healthcare centers so that would no need to wait long times for the radiologists to interpret the images.
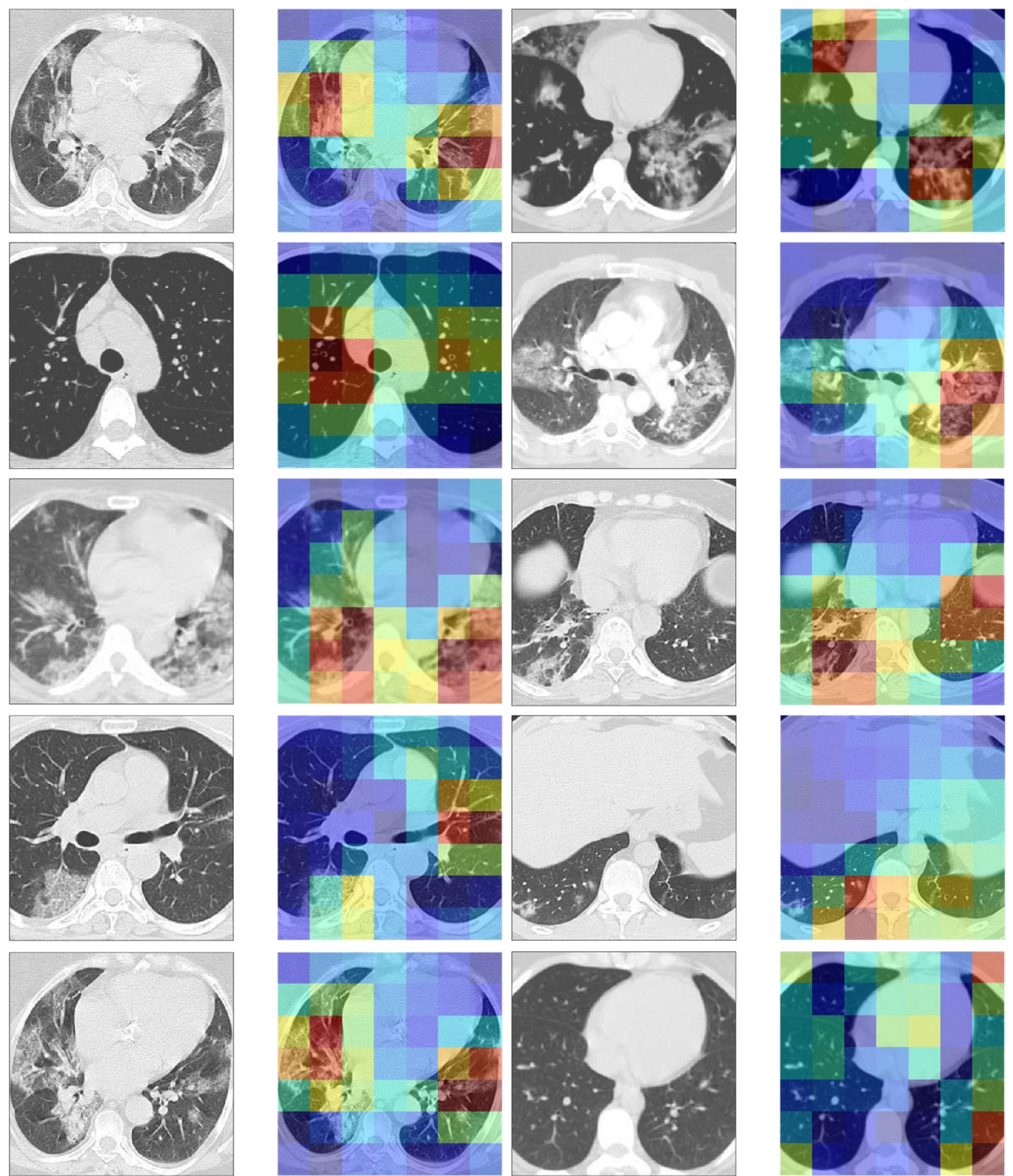

Figure 4. The illustration of original COVID-19 positive CT scans and calculated activation maps of ResNet50 architecture 


\section{Conclusion and Comment}

The rapid diagnosis and triaging are vital for the control of infectious diseases, specifically COVID-19. There is urgent requirement for alternative effective and timely diagnosis methods that can be utilized by front-line health care personal to quick and accurate diagnosis of COVID-19. Bearing this in mind, in this study, a transfer learning based CNN model was developed to screen COVID-19 infected patients from chest CT images. The performance evolution results of the model have pointed out the proof of principle that proposed model can accurately extract hallmarks of COVID-19 from chest CT scans to diagnose the COVID-19 pneumonia. As a result of training steps, it was concluded that only fully connected layers were not enough to re-train. Thus, convolutional layers were also re-trained and the performances of the networks were increased with fine-tuning process. ResNet50 was identified as the most suitable CNN architecture among fine-tuned architectures. In the last step of the model development, an activation map was created by implementing the Grad-CAM algorithm, and radiologists were informed about which points artificial intelligence focused on the graph. Furthermore, it is thought that comparing different architectures and choosing suitable architecture will make a great contribution to the literature. In essence, it can be speculated that by utilizing developed model in the healthcare system the diagnosis time can significantly shorten so the disease can effectively be controlled and the possible spreading risk of virus can be prevented. Moreover, in this way, the work-load of the experts in the field can significantly be reduced.

\section{Author Statement}

Onur Karaman: Methodology, Software, Validation, Original Draft Writing, Investigation

\section{Acknowledgment}

The author would like to thank the anonymous referees for helpful suggestions that improved the paper.

\section{Conflict of Interest}

As the author of this study, I declare that I do not have any conflict of interest statement.

\section{Ethics Committee Approval and Informed Consent}

As the author of this study, I declare that I do not have any ethics committee approval and/or informed consent statement.

\section{References}

[1] C. Butt, J. Gill, D. Chun, and B. A. Babu, "Deep learning system to screen coronavirus disease 2019 pneumonia," Appl. Intell., 1-7, 2020.

[2] T. Singhal, "A review of coronavirus disease-2019 (COVID-19)," Indian J Pediatr, 1-6, 2020.

[3] S. H. Ebrahim, Q. A. Ahmed, E. Gozzer, P. Schlagenhauf, and Z. A. Memish, "Covid-19 and community mitigation strategies in a pandemic," BMJ, 2020.

[4] Coronavirus disease 2019 (COVID-19) Situation Report - 51, WHO, [Online].Available: https://www.who.int/docs/default-source/coronaviruse/situation-reports/20200311-sitrep-51-covid 19.pdf?sfvrsn=1ba62e57_10. Accessed 07 June 2020.

[5] Resource estimation for contact tracing, quarantine and monitoring activities for COVID-19 cases in the EU/EEA European Centre for Disease Prevention and Control (ECDC):Stockholm, [Online]. Available:https://www.ecdc.europa.eu/en/publications-data/resource-estimation-contact-tracingquarantine-and-monitoring-activities-covid-19.pdf. Accessed 07 June 2020

[6] L. Wang and A. Wong, "COVID-Net: A tailored deep convolutional neural network design for detection of COVID-19 cases from chest radiography images," arXiv 2020 preprint arXiv:2003.09871. 
[7] T. Ai, Z. Yang, H. Hou, C. Zhan, C. Chen, W. Lv, Q. Tao, Z. Sun, and L. Xia, "Correlation of chest CT and RT-PCR testing in coronavirus disease 2019 (COVID-19) in China: a report of 1014 cases," Radiology, 200642, 2020.

[8] N. Chen, M. Zhou , X. Dong, J. Qu, F. Gong, Y. Han, et al., "Epidemiological and clinical characteristics of 99 cases of 2019 novel coronavirus pneumonia in Wuhan, China: a descriptive study," The Lancet, 395, 507-513, 2020.

[9] S. Wang, B. Kang, J. Ma , X. Zeng , M. Xiao, J. Guo, et al., "A deep learning algorithm using CT images to screen for Corona Virus Disease (COVID-19)," MedRxiv, 2020.

[10] J. P. Kanne, B. P. Little, J.H. Chung, B.M. Elicker, L.H. Ketai, "Essentials for radiologists on COVID-19: an update-radiology scientific expert panel," Radiology, 2020.

[11] J. Zhang, Y. Xie, Y. Li, C. Shen, and Y. Xia, "COVID-19 screening on chest X-ray images using deep learning based anomaly detection," arXiv 2020,preprint arXiv:2003.12338.

[12] O. Gozes , M. Frid-Adar , H. Greenspan , P. D. Browning, et al., "Rapid AI development cycle for the coronavirus (COVID-19) pandemic: Initial results for automated detection \& patient monitoring using deep learning CT image analysis," arXiv 2020,preprint arXiv:2003.05037.

[13] T. Ozturk, M. Talo, E. A. Yildirim, U. B. Baloglu, O. Yildirim, and U. R. Acharya, "Automated detection of COVID-19 cases using deep neural networks with X-ray images," Comput. Biol., $103792,2020$.

[14] E. Soares, P. Angelov, S. Biaso, M. H. Froes, D. K. Abe, "SARS-CoV-2 CT-scan dataset: A large dataset of real patients CT scans for SARS-CoV-2 identification," medRxiv, 2020.

[15] A. Bernheim , X. Mei , M. Huang, Y. Yang , Z. A. Fayad , N. Zhang, et al., "Chest CT findings in coronavirus disease-19 (COVID-19): relationship to duration of infection," Radiology, 200463, 2020.

[16] W. Zhao, Z. Zhong, X. Xie, Q. Yu, and J. Liu, "Relation between chest CT findings and clinical conditions of coronavirus disease (COVID-19) pneumonia: a multicenter study," Am J Roentgenol, 214(5), 1072-1077, 2020.

[17] F. Shan , Y. Gao, J. Wang, W. Shi, N. Shi, et al., "Lung infection quantification of COVID-19 in CT images with deep learning," arXiv 2020,preprint arXiv:2003.04655.

[18] R. M. Elavarasan and R. Pugazhendhi, "Restructured society and environment: A review on potential technological strategies to control the COVID-19 pandemic," Sci Total Environ., 138858, 2020.

[19] Fastai [Online].Available: https://www.fast.ai/ Accessed 07 June 2020.

[20] K. He, X. Zhang, S. Ren, and J. Sun, "Deep residual learning for image recognition," in Proc. IEEE conference on computer vision and pattern recognition, 2016, 770-778.

[21] K. Simonyan and A. Zisserman, "Very deep convolutional networks for large-scale image recognition," arXiv 2014 Sep: preprint arXiv:1409.1556.

[22] F. N. Iandola, S. Han, M.W. Moskewicz, K. Ashraf, W. J. Dally, and K. Keutzer, "SqueezeNet: AlexNet-level accuracy with 50x fewer parameters and<0.5 MB model size," arXiv 2016, preprint arXiv:1602.07360.

[23] ImageNET [Online].Available: http://www.image-net.org/papers/imagenet_cvpr09.bi Accessed 07 June 2020 .

[24] I. Loshchilov and F. Hutter, "SGDR: Stochastic gradient descent with warm restarts," arXiv 2016, preprint arXiv:1608.03983.

[25] A. Holzinger, G. Langs, H. Denk, K. Zatloukal, and H. Müller, "Causability and explainability of artificial intelligence in medicine," Wires Data Min Knowl., 9, 1312, 2019.

[26] R. R. Selvaraju, M. Cogswell, A. Das, R. Vedantam, D. Parikh, and D. Batra, "Grad-CAM: Visual explanations from deep networks via gradient-based localization," in Proc. IEEE international conference on computer vision, 2017, 618-626.

[27] Y. Bengio, A. Courville, and P. Vincent, "Representation learning: A review and new perspectives," IEEE Trans Pattern Anal Mach Intell, 35, 1798-1828, 2013.

[28] A. Mahendran and A. Vedaldi, "Salient deconvolutional networks," in Proc. Computer Vision - ECC V, 2016, 120-135.

[29] T. Fawcett, “An introduction to ROC analysis,” Pattern Recognit Lett., 27, 861-864, 2006. 